ARTICLE

https://doi.org/10.1038/s41467-018-08116-7

\title{
The HisCl1 histamine receptor acts in photoreceptors to synchronize Drosophila behavioral rhythms with light-dark cycles
}

Faredin Alejevski ${ }^{1}$, Alexandra Saint-Charles ${ }^{1,2}$, Christine Michard-Vanhée ${ }^{1}$, Béatrice Martin ${ }^{1}$, Sonya Galant ${ }^{1}$, Daniel Vasiliauskas ${ }^{1} \&$ François Rouyer (i) ${ }^{1}$

In Drosophila, the clock that controls rest-activity rhythms synchronizes with light-dark cycles through either the blue-light sensitive cryptochrome (Cry) located in most clock neurons, or rhodopsin-expressing histaminergic photoreceptors. Here we show that, in the absence of Cry, each of the two histamine receptors Ort and HisCl1 contribute to entrain the clock whereas no entrainment occurs in the absence of the two receptors. In contrast to Ort, $\mathrm{His} \mathrm{Cl} 1$ does not restore entrainment when expressed in the optic lobe interneurons. Indeed, $\mathrm{His} C \mathrm{Cl}$ is expressed in wild-type photoreceptors and entrainment is strongly impaired in flies with photoreceptors mutant for His Cl1. Rescuing HisCl1 expression in the Rh6-expressing photoreceptors restores entrainment but it does not in other photoreceptors, which send histaminergic inputs to Rh6-expressing photoreceptors. Our results thus show that Rh6expressing neurons contribute to circadian entrainment as both photoreceptors and interneurons, recalling the dual function of melanopsin-expressing ganglion cells in the mammalian retina.

\footnotetext{
${ }^{1}$ Institut des Neurosciences Paris-Saclay, Univ. Paris Sud, CNRS, Université Paris-Saclay, 91190 Gif-sur-Yvette, France. ${ }^{2}$ Present address: Institut de la Vision, Univ. P. \& M. Curie, INSERM, CNRS, Sorbonne Université, Paris 75012, France. These authors contributed equally: Faredin Alejevski, Alexandra Saint-Charles. Correspondence and requests for materials should be addressed to F.R. (email: rouyer@inaf.cnrs-gif.fr)
} 
$\mathrm{T}$ he Drosophila sleep-wake rhythms are controlled by a brain circadian clock that includes about 150 clock neurons $^{1}$. Light synchronizes the clock neuronal network through cell-autonomous and non-cell-autonomous light input pathways ${ }^{2,3}$. Cry is a blue-light sensitive photoreceptor protein that is expressed in most clock neurons ${ }^{4-8}$. In the absence of Cry, flies do not phase-shift their behavioral rhythms in response to a short light pulse but still synchronize to light-dark (LD) cycles ${ }^{4}$. Only flies devoid of both Cry and rhodopsin-expressing photoreceptors fail to entrain to $\mathrm{LD}$ cycles $^{2,7}$. Six different rhodopsins (Rhs) have been characterized in the Drosophila photoreceptive structures, which include the compound eye, the HofbauerBuchner (H-B) eyelet, and ocelli. The compound eye strongly contributes to circadian photoreception, whereas a modest contribution appears to be brought by the H-B eyelet and the ocelli ${ }^{3,9-11}$. A circadian function has been recently associated with the yet poorly characterized rhodopsin 7 , although its exact contribution and localization in the brain and/or the eye remains controversial ${ }^{12-14}$. In addition to entrainment, the visual system controls other features of the clock neuron network by conveying light information to either promote or inhibit the behavioral output of specific clock neuron subsets ${ }^{15-17}$.

The compound eye includes about 800-unit eyes (ommatidia), each of which contains eight photoreceptors. The six Rh1expressing outer photoreceptors (R1-6) are involved in motion detection and project to the lamina neuropile of the optic lobe. The two inner photoreceptors (R7-8) are important for color detection and project to the medulla. They express four different rhodopsins and thus define two types of ommatidia: "pale" (p) ommatidia (30\%) include a Rh3-expressing R7 and a Rh5expressing R8, whereas "yellow" (y) ommatidia (70\%) include a Rh4-expressing R7 and a Rh6-expressing R8 ${ }^{18,19}$. Each extraretinal H-B eyelet contains four Rh6-expressing photoreceptors that project to the accessory medulla, in the vicinity of key pacemaker neurons, the ventral lateral neurons (LNvs) that produce the pigment-dispersing factor (PDF) neuropeptide ${ }^{9,20-24}$. Each of the three ocelli contains about 80 photoreceptors that express $\mathrm{Rh} 2^{25}$. The Drosophila rhodopsins cover a wide range of wavelengths from $300 \mathrm{~nm}$ to $600 \mathrm{~nm}^{18,19}$, with only Rh1 and Rh6 being sensitive to red light ${ }^{26}$.

Rhodopsin-dependent circadian entrainment involves two downstream signaling pathways, the canonical one that relies on the phospholipase $\mathrm{C}$ encoded by the no receptor potential $A$ gene (norpA $)^{27,28}$ or an unknown pathway that does not contribute in very low light levels ${ }^{4}$. All but $\mathrm{Rh} 2$ - and $\mathrm{Rh} 5$ - expressing photoreceptors support synchronization in very low light ${ }^{29}$, and at least $\mathrm{Rh} 1, \mathrm{Rh} 5$, and Rh6 can signal through the NorpA-independent pathway ${ }^{30,31}$. Photoreceptors of the compound eye are histaminergic ${ }^{27,28}$ but the H-B eyelet expresses both histamine and acetylcholine $e^{32,33,22}$. Although the two neurotransmitters might contribute to circadian entrainment ${ }^{34,35}$, flies devoid of Cry and histidine decarboxylase do not synchronize their rest-activity rhythms with LD cycles ${ }^{10}$. This suggests that besides Cry, there is no histamine-independent pathway to entrain the clock.

Two genes encoding histamine-gated chloride channels, ora transientless (ort) and Histamine-gated chloride channel subunit 1 (HisCl1), have been identified in Drosophila ${ }^{36-39}$. The ort-null mutants are visually blind $\mathrm{d}^{40,41}$ and their electroretinograms have no $\mathrm{ON}$ and $\mathrm{OFF}$ transients ${ }^{42}$. In contrast, $\mathrm{His} \mathrm{Cl} 1$ mutants show increased OFF transients, whereas slower responses were observed in the postsynaptic laminar monopolar cells $\mathrm{s}^{42}$. Based on transcriptional reporters, ort expression in the optic lobes was observed in neurons of both the lamina and medulla/lobula neuropils ${ }^{41-44}$. Based on reporter gene expression, HisCll was localized in glial cells of the lamina ${ }^{41,42}$. However, recent work reported expression in photoreceptors, in particular in the R7 and
R8 inner photoreceptor subtypes ${ }^{45-47}$. Indeed, Ort and HisCl1 support color opponency between the two subtypes of "inner" photoreceptors, the ultraviolet (UV)-sensitive R7 and non-UV-sensitive R8, with HisCl1 and Ort mediating direct and indirect inhibition, respectively ${ }^{47}$. The histaminergic pathways that are involved in circadian entrainment are unknown and are the subject of the present study. Our results show that both Ort and HisCll define two different pathways for circadian entrainment. Whereas Ort contributes through its expression in the interneurons of the optic lobe, HisCl1 mostly contributes through its expression in the Rh6-expressing retinal photoreceptors. The work thus reveals that Rh6-expressing neurons contribute to light-mediated entrainment as both photoreceptors and interneurons.

\section{Results}

Ort and HisCl1 both support synchronization with LD cycles. We asked whether Ort and HisCll mediate circadian entrainment. Like wild-type flies and $\mathrm{cry}^{0}$ mutants, $\mathrm{HisCl}^{134}$ ort $^{1}$ double mutants resynchronized their rest-activity rhythms when the LD cycle is either advanced or delayed by several hours (Fig. 1a, Supplementary Fig. 1). In contrast, $\mathrm{cry}^{02} \mathrm{HisCl1}^{134}$ ort $^{1}$ triple mutants (hereafter $\mathrm{CHO}$ mutants) did not synchronize (Fig. 1a), indicating that at least one of the two chloride channels is required in the absence of Cry. We thus tested the contribution of each of the two histamine-gated channels. Flies devoid of either Cry and HisCl1 or Cry and Ort efficiently synchronized (Fig. 1a, Supplementary Fig. 1, Supplementary Table 1,2), showing that HisCl1 or Ort can each mediate Cry-independent entrainment of activity rhythms. Finally, we asked whether the two histamine receptors contributed to both the canonical and NorpA- independent transduction pathways ${ }^{4}$. As expected, $c r y^{0}$ norp $A^{24}$ double mutants did not synchronize with low light LD cycles (see Methods), but $c r y^{0}$ mutants with either only HisCl1 or only Ort supported synchronization through the NorpA-dependent pathway (Supplementary Fig. 1). In the absence of NorpA and Cry, flies with one or the other receptor synchronized with high light LD cycles (Supplementary Fig. 1). Each of the two histamine receptors can thus mediate synchronization through NorpAdependent and NorpA-independent pathways. We conclude that, in contrast to vision, circadian photoreception can thus be independently supported by Ort and HisCl1.

We tested the ability of HisCl1 and Ort to mediate circadian entrainment through inputs from either the Rh1 photoreceptors that project to the lamina or the Rh6 photoreceptors, which include retinal neurons that project to the medulla and $\mathrm{H}-\mathrm{B}$ eyelet neurons that project to the accessory medulla. Since circadian entrainment to red light $\mathrm{LD}$ cycles (hereafter $\mathrm{RD}$ cycles) requires either Rh1 or $\mathrm{Rh}^{26}{ }^{26}$, we used a shifted RD cycle protocol to functionally isolate these two rhodopsins. The four possible combinations of histamine receptor and rhodopsin mutants synchronized with $\mathrm{RD}$ cycles, although flies with only $\mathrm{Rh} 1$ and HisCl1 did it more slowly (Fig. 1b, Supplementary Fig. 1, Supplementary Tables 1,2). HisCl1 and Ort could thus each respond to inputs from Rh1-expressing outer and Rh6-expressing R8 inner photoreceptors, suggesting that HisCl1 is not only expressed in the lamina.

Ort and HisCl1 define different light input pathways. To identify the circuits between photoreceptors and clock neurons, we asked whether targeting expression of HisCll or Ort in different neuronal subsets of $\mathrm{CHO}$ mutants would restore entrainment. We first used the tim-gal4 driver, whose expression in the head includes brain clock neurons, photoreceptors, and other neurons and glial cells, in particular in the optic lobes ${ }^{48}$. 
a

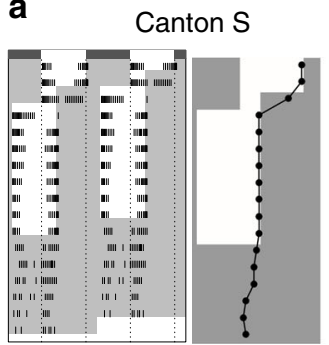

Wild type

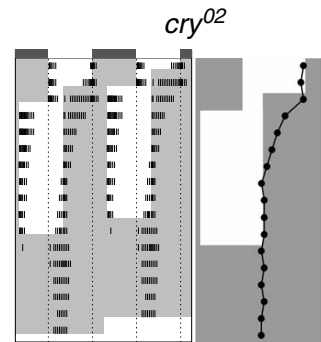

No Cry

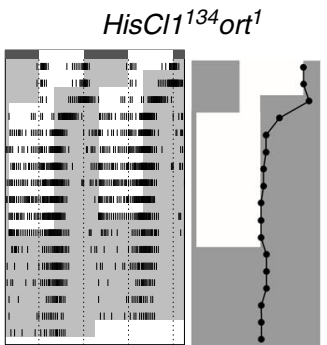

No HisCl1, no Ort b

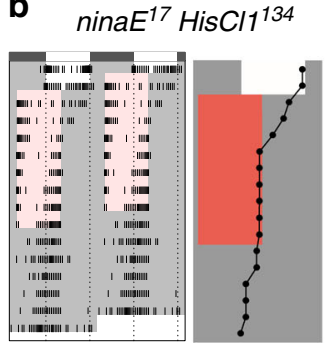

No Rh1, no HisCl1

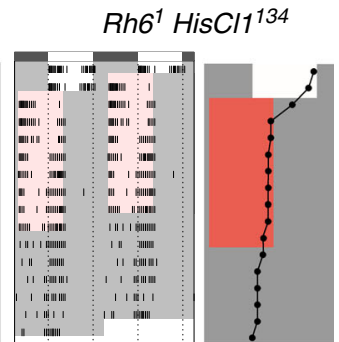

No Rh6, no HisCl1

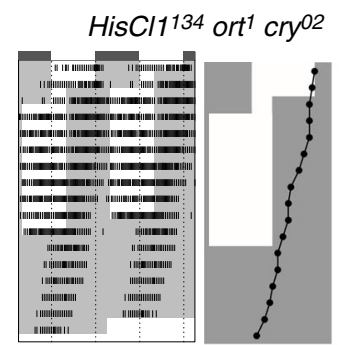

No HisCl1, no Ort, no Cry

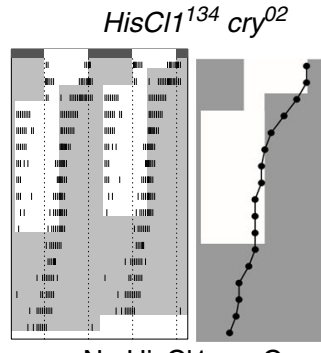

No HisCl1, no Cry

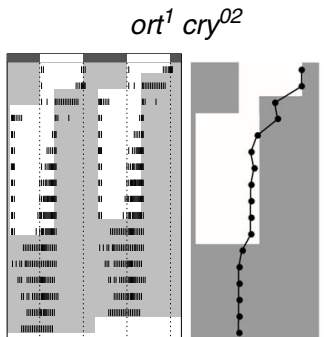

No Ort, no Cry

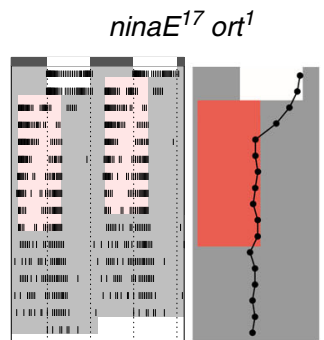

No Rh1, no Ort

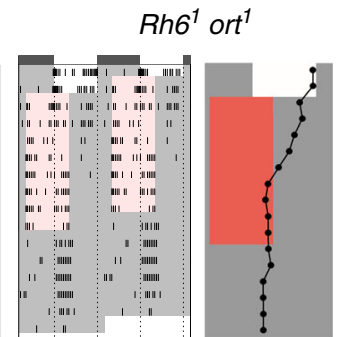

No Rh6, no Ort

Fig. $1 \mathrm{His} C l 1$ and Ort histamine receptors can each transmit light input from Rh1- and Rh6-expressing PRs. a, b Average double plots of locomotor activity (actograms) of flies exposed to an $8 \mathrm{~h}$ advance of the LD/RD cycle, with corresponding phase plots (see Methods). Flies were initially entrained with both $\mathrm{LD}$ and temperature cycles (TC: $\left.25-20^{\circ} \mathrm{C}\right)$. a $\mathrm{T}^{\circ} \mathrm{C}$ was kept constant $\left(25^{\circ} \mathrm{C}\right)$ from the beginning of the day 3 light phase until the end of the experiment and the day 3 light phase was shortened by $8 \mathrm{~h}$. Thus, flies were exposed to a novel advanced LD regime for 8 cycles followed by constant darkness ( 6 DD cycles). (Top) Wild-type flies (left) as well as flies with no Cry (center) or no histamine receptors (right) synchronize with phase advanced LD cycles. (Bottom) Flies with no Cry and no histamine receptors do not synchronize (left), whereas flies with no cry and only Ort (center) or only HisCl1 (right) do synchronize. $\mathbf{b} \mathrm{T}^{\circ} \mathrm{C}$ was kept constant $\left(25^{\circ} \mathrm{C}\right)$ from the beginning of the day 2 light phase until the end of the experiment. Between days 2 and 3 , the dark phase was shortened by $8 \mathrm{~h}$ and red light was used for the advanced light phase. Thus, flies were exposed to a novel advanced RD (red light) regime for 8 cycles followed by 6 DD cycles. Each of the four genotypes with only one histamine receptor and either Rh1 or Rh6 rhodopsin synchronizes with advanced RD cycles. Bars above actograms indicate the initial LD/TC cycles (black: lights-off, $20^{\circ} \mathrm{C}$; white: lights-on, $25^{\circ}$ ). White or red areas indicate the light phase of the cycle with white or red light respectively, and gray areas the dark phase of the cycle. Dots of the phase plots indicate the peak value of evening activity in LD/RD cycles and of activity in DD. $n$ is the number of flies. Some genotypes (e.g., CHO, in (a) bottom left) have a free-running period that is slightly shorter than $24 \mathrm{~h}$, which results in gradual advancement of the activity peak in DD or in LD/RD (also see other figures) when not entrained

Expression of either HisCl1 or ort under tim-gal4 control restored synchronization (Fig. 2a). Expression of either histamine receptor in glial cells with the repo driver ${ }^{49}$ did not rescue the $\mathrm{CHO}$ phenotype (Supplementary Fig. 2), supporting the idea that neuronal expression is required for the circadian function of both Ort and HisCl1. Further, HisCl1-gal4-driven glial expression ${ }^{41,42}$ did not allow HisCl1 or Ort to restore synchronization (Supplementary Fig. 2), suggesting that HisCl1-gal4 does not recapitulate endogenous $\mathrm{His} \mathrm{Cl}$ expression. Driving ort expression with the ort $^{c-4}$-gal4 construct that includes the four blocks of conserved sequences identified in the non-transcribed ort genomic region ${ }^{41}$ allowed synchronization (Fig. 2b, Supplementary Tables 1, 2). However, ort ${ }^{c 1-4}$-gal4-driven HisCl1 expression did not (Fig. 2b, Supplementary Tables 1,2), indicating that HisCll could not functionally replace Ort in ort-expressing neurons. Thus, Ort and HisCl1 appear to define two different neuronal pathways for the synchronization of rest-activity rhythms.

The small and large LNvs send processes in the accessory medulla and the large LNvs also extensively arborize in the medulla, suggesting that PDF cells could receive direct inputs from inner photoreceptors and/or eyelet $9,23,34,35,50$. We thus targeted HisCll or Ort expression to the clock neurons of $\mathrm{CHO}$ mutants and tested entrainment. Neither HisCll nor Ort could restore synchronization when expressed in the PDF neurons or even with the Clk:gal4(6/1 $)^{51}$ that is expressed in most clock neurons, including all lateral (LNvs, dorsal lateral neurons, and lateral posterior neurons) and dorsal neurons (DN1, DN2, DN3 subsets) (Fig. 2c, Supplementary Fig. 2). Our data indicate that direct histaminergic inputs from the photoreceptors to the clock neurons are not sufficient for circadian entrainment and support an indirect connection through HisCl1- and Ortexpressing interneurons.

The failure of HisCl1 to allow synchronization when expressed in Ort-expressing neurons prompted us to look for other types of neurons that potentially express HisCl1. Surprisingly, targeting HisCl1 to all photoreceptors with GMR-gal4 rescued the entrainment of $\mathrm{CHO}$ mutants (Fig. 2d, Supplementary Table 1,2). Furthermore, rescue was no longer observed in tim $>\mathrm{His} \mathrm{Cll}$, CHO flies when $\mathrm{His} \mathrm{Cl} 1$ expression in photoreceptors was blocked in the photoreceptors by concomitantly expressing the Gal4 inhibitor Gal80 under GMR control (Fig. 2d, Supplementary Table 1,2$)$. This raised the intriguing possibility that HisCll circadian function occurs in photoreceptor cells.

HisCl1, but not Ort, is expressed in photoreceptors. Transcriptome analysis indicated that $\mathrm{HisCl1}$ but not ort was expressed in photoreceptors ${ }^{45,46}$. In agreement with previous studies $^{41-43}$, we could not detect any photoreceptor-localized expression of the different ort-gal4 drivers (not shown). The published anti-HisCl1 antibody ${ }^{3} 3$ only produced non-specific labeling in our hands and a HisCl1-gal4 construct showed expression only in the optic lobe, essentially in lamina cells as described previously ${ }^{41}$. However, by using two copies of a second HisCl1-gal4 construct ${ }^{42}$, we could faintly detect some inner retinal photoreceptors and the eyelet, in addition to the lamina cells (Fig. 3a-d). The expression of $\mathrm{HisCl1}$ in photoreceptors was also faintly detected by a HisCl1::egfp protein fusion genomic 

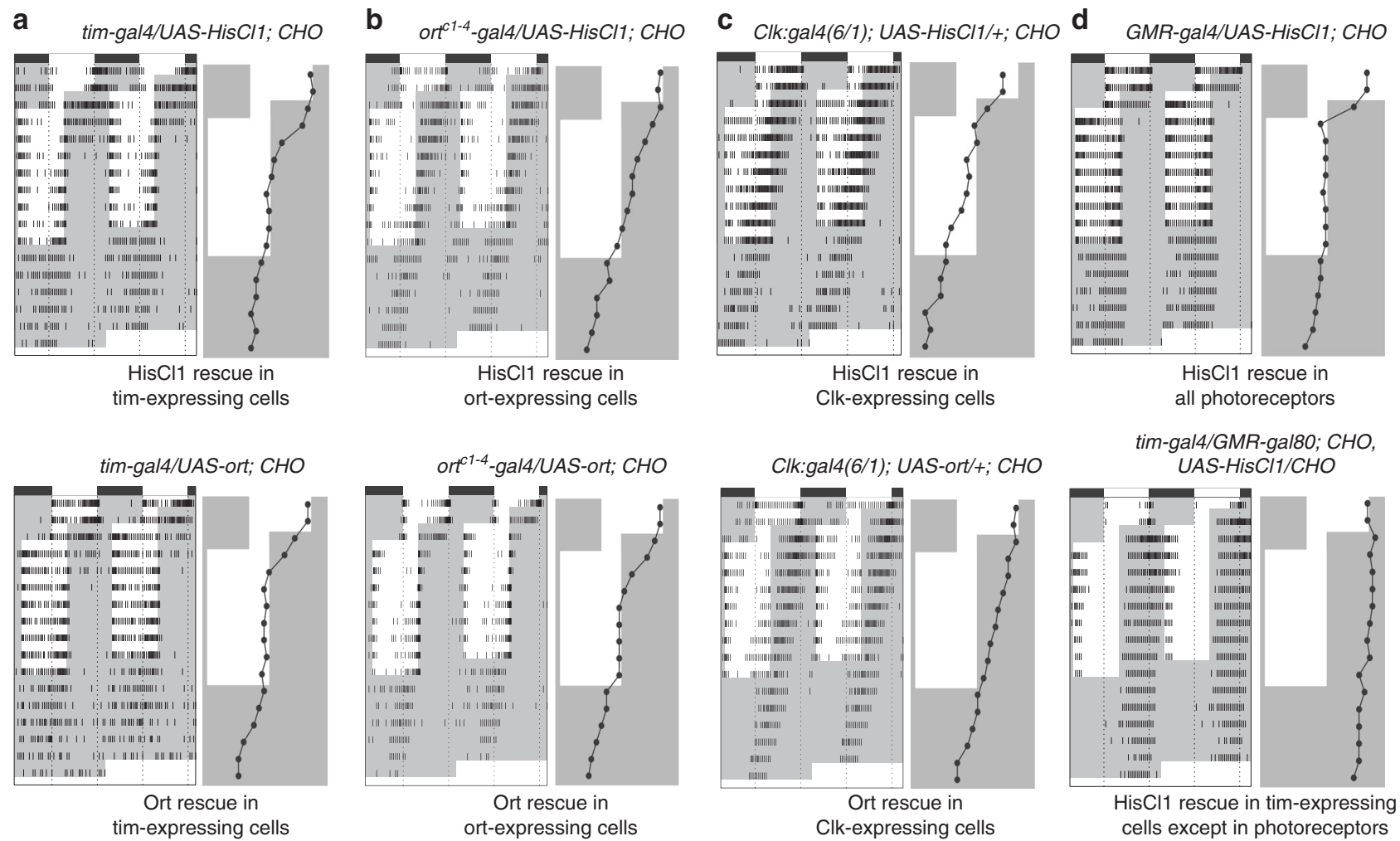

Fig. 2 Ort and HisCl1 define different neuronal pathways for clock synchronization. Actograms and phase plots of flies with expression of the two histamine receptors in the triple mutant ( $\mathrm{His} \mathrm{Cl} 7^{134}$ ort $^{1} \mathrm{cry}^{02}{ }_{-} \mathrm{CHO}$ ) genetic background using different gal4 drivers. The experimental design is as described in Fig. 1 legend. a Expression of either HisCl1 (top) or ort (bottom) in tim-expressing cells rescues entrainment. b HisCll cannot rescue entrainment when expressed in ort-expressing neurons (top), whereas ort can (bottom). c Neither HisCl1 (top) nor ort (bottom) rescues entrainment when expressed in most of the clock neurons. $\mathbf{d}$ Entrainment is rescued by His $\mathrm{Cl}$ expression in photoreceptors (top) and is not rescued by HisCl1 expression in tim-expressing cells when expression in the photoreceptors is blocked by the Gal4 inhibitor Gal80 (GMR-gal80) (bottom)

construct (see Methods), which labeled the cell bodies of both a fraction of R7 and R8 inner retinal photoreceptors and eyelet photoreceptors (Fig. 3e-g). HisCl1::egfp expression was recently reported in R7 and R8 inner photoreceptors ${ }^{47}$. To confirm HisCl1 reporter gene expression data, we addressed the retinal expression of the ort and/or HisCl1 genes with a new sensitive in situ hybridization technique (see Methods). Indeed, HisCl1 messenger RNA (mRNA) was present at low levels in photoreceptors of wild-type flies, including Rh6-expressing R8 cells, whereas no transcripts were detected in Hiscl1 ${ }^{134}$ mutants (Fig. $3 \mathrm{~h}-\mathrm{k}$ ) or with a negative control probe (not shown). In contrast, ort expression was observed in interneurons but not in photoreceptors (Fig. 31, m).

HisCl1 circadian role is in Rh6-expressing photoreceptors. The expression data suggested that HisCl1 circadian function could map to inner photoreceptors of the retina and/or eyelet. Among inner photoreceptors, R7 cells express Rh3 or Rh4, whereas R8 cells express Rh5 or Rh6 ${ }^{18,19,52}$. We thus restored HisCl1 in $\mathrm{CHO}$ mutants with different types of $R h$-gal4 constructs. A fast synchronization with the shifted LD cycle was observed with Rh6gal4, which was lost in the presence of GMR-gal80 (Fig. 4a, Supplementary Tables 1,2$)$. In contrast, none of the other Rh-gal4 rescued entrainment (Supplementary Fig. 3, Supplementary Tables 1, 2), showing that HisCl1 acts specifically in Rh6expressing photoreceptors. Of course, we cannot exclude a possibility that synchronization rescue could occur over longer exposure to the $\mathrm{LD} / \mathrm{RD}$ cycle. We also observed that this new role of Rh6 cells could mediate circadian entrainment through both
NorpA-dependent photoreception in low light and NorpAindependent photoreception in high light (Supplementary Fig. 3, Supplementary Tables 1, 2). Surprisingly, expressing Ort in Rh6 cells also rescued entrainment (Supplementary Fig. 3, Supplementary Tables 1, 2), indicating that Ort can replace HisCl1 function in the photoreceptors, whereas HisCl1 could not replace Ort in the ort-expressing interneurons of the optic lobe (see Fig. 2a).

What is the specific contribution of HisCl1 function in $\mathrm{Rh} 6$ photoreceptors to circadian entrainment? To address this question, we first downregulated HisCl1 in Rh6 cells, with other putative sites of HisCll expression being not affected. HisCl1 RNA interference (RNAi) was expressed in a $c r y^{0}$ ort $^{1}$ double mutant background and the flies were tested in $\mathrm{RD}$ cycles to restrict light inputs to Rh1 and Rh6 cells. HisCl1 RNAi flies did synchronize with the new light regime but substantially slower than the control flies, supporting a contribution of $\mathrm{HisCl} 1$ expression in Rh6 photoreceptors to entrainment (Fig. 4b, Supplementary Fig. 4 and Supplementary Tables 1, 2). The fact that HisCl1 RNAi flies still synchronize, although slowly, could result from an incomplete loss of HisCl1 function in $\mathrm{Rh} 6$ photoreceptors and/or from the existence of a photoreceptorindependent role of HisCl1. A strong contribution of HisCl1 in photoreceptors was confirmed by testing flies that were mutant for HisCl1 in whole eye clones (see Methods). The synchronization with advanced LD cycles was strongly slowed down (Fig. 4c, Supplementary Fig. 4, Supplementary Table 1), although our statistical test failed to capture a significant difference with controls in the last 5 days of the shifted LD cycle (Supplementary Table 2). However, flies with HisCl1 ${ }^{134}$ mutant eyes failed to 

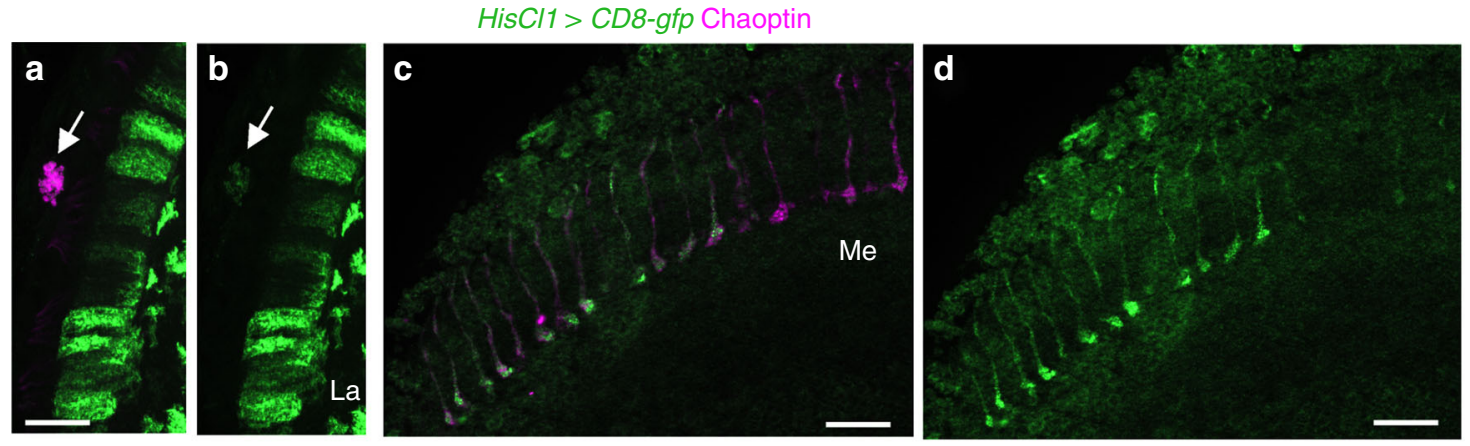

HisCl1::egfp ey-rfp
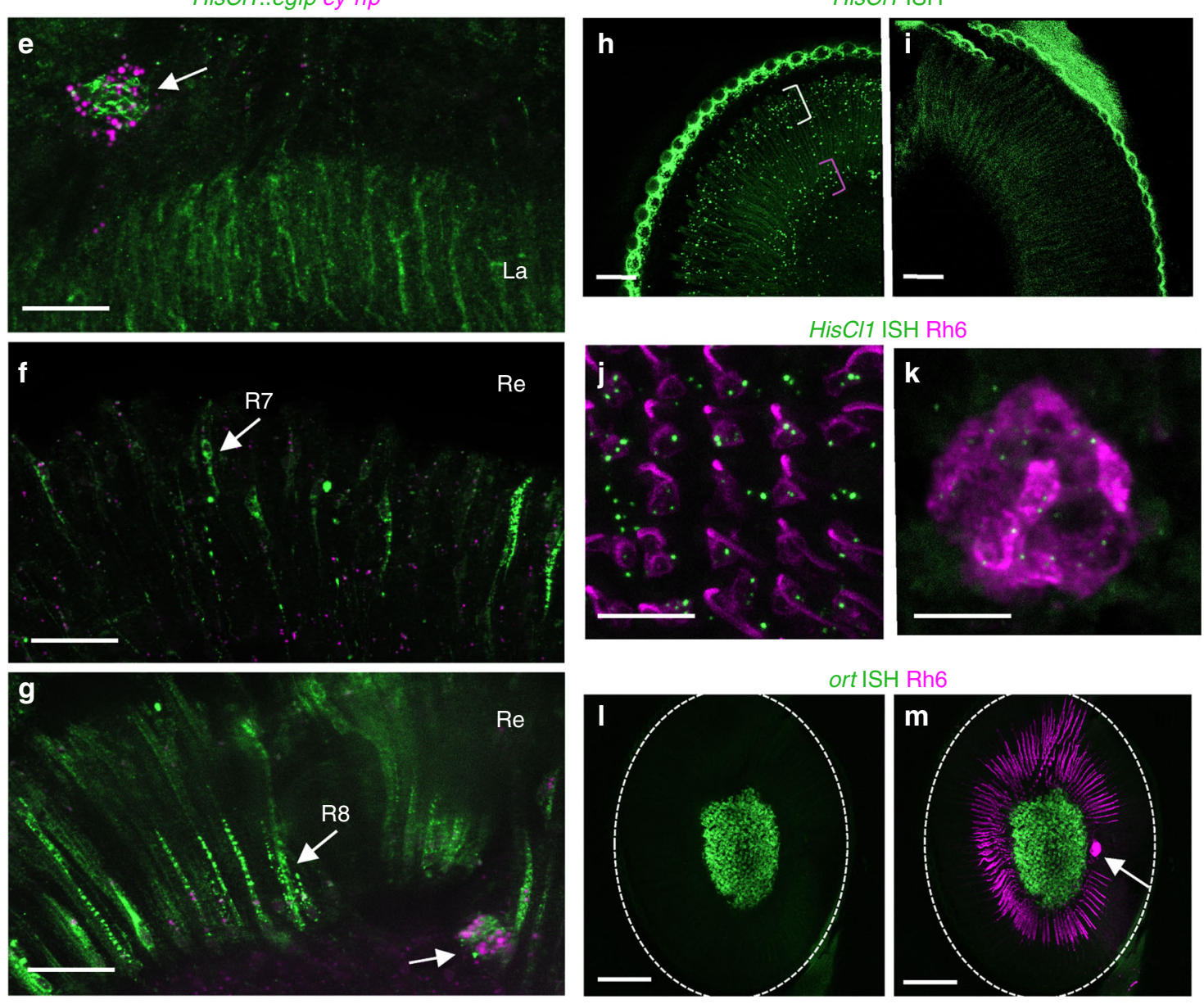

Fig. $3 \mathrm{HisCl1}$ but not Ort is expressed in photoreceptors. a-d Expression of HisCl1-gal4 UAS-mCD8-gfp, detected with anti-GFP antibody (green); photoreceptors are marked with anti-Chaoptin antibody (red). HisCl1 expression is detected in the lamina epithelial cells and very faintly in the eyelet (a, b). Weak labeling is observed in some R7 and R8 photoreceptors axons in the medulla (c, d). e-g Expression of GFP-tagged His Cl1 protein expressed from the His Cl1 promoter detected with anti-GFP antibody (green); photoreceptors are marked with ey-rfp from the same fosmid construct (red). HisCl1::GFP is detected in the lamina (e), H-B eyelet $(\mathbf{e}, \mathbf{g})$, and both in the R7 (f) and R8 (g) photoreceptors, identified by their respective position in the retina (arrows), upper layer for R7 and lower layer for R8. h-m $\mathrm{His} C l 1$ (h-k) and ort (I, $\mathbf{m})$ mRNA expression (blue) visualized by RNAscope in situ hybridization (ISH) (see Methods). Anti-Rh6 antibody (red) labels yR8 and eyelet photoreceptors in (j, $\mathbf{k}, \mathbf{m})$. HisCl1 mRNA in the retina appears as puncta primarily in two layers: proximally, near the R8 photoreceptor nuclei (h pink bracket) and distally, near the R1-7 photoreceptor and other retinal cell nuclei (h yellow bracket). No puncta were detected in the His $\mathrm{Cl} 7^{134}$ mutant (i). In the R8 photoreceptor layer HisCl1 mRNA is expressed in the Rh6-positive (red) yR8 retinal photoreceptors, as well as in the surrounding cells that likely include Rh5-expressing pR8 photoreceptors (j). HisCl1 mRNA is also present in the Rh6expressing H-B eyelet (k). ort mRNA is expressed in the lamina but not in the retina $(\mathbf{I}, \mathbf{m})$. In particular, ort is undetectable in the Rh6-expressing cells (red in $\mathbf{m}$ ) both in the retina (outlined in $\mathbf{I}, \mathbf{m})$ and in the eyelet. In (h, i), the retinas are surrounded by the autofluorescent cuticle. Images in (h-m) are maximal projections of short confocal stacks (thickness: $10(\mathbf{h}, \mathbf{i}), 12(\mathbf{j}, \mathbf{k}), 13.5(\mathbf{I}-\mathbf{m}) \mu \mathrm{m})$. La lamina, Me medulla, Re retina. White arrows point to the H-B eyelet. Scale bars represent $20(\mathbf{a}-\mathbf{i}), 10(\mathbf{j}, \mathbf{k})$, and $50(\mathbf{I}, \mathbf{m}) \mu \mathrm{m}$ 


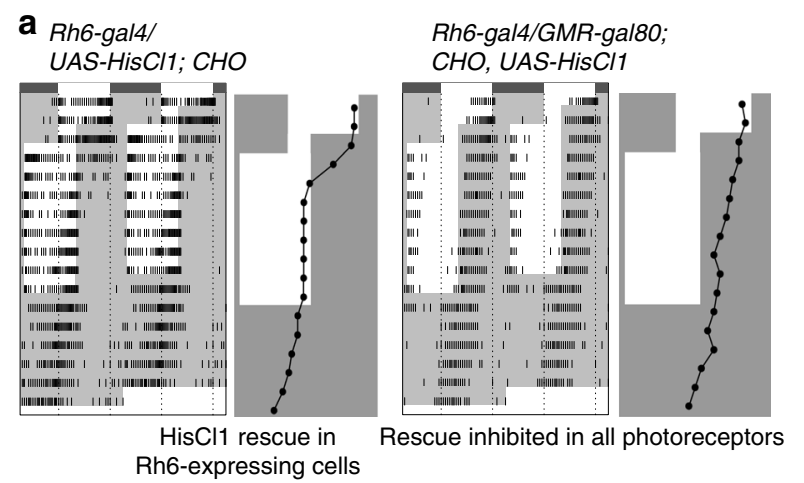

C
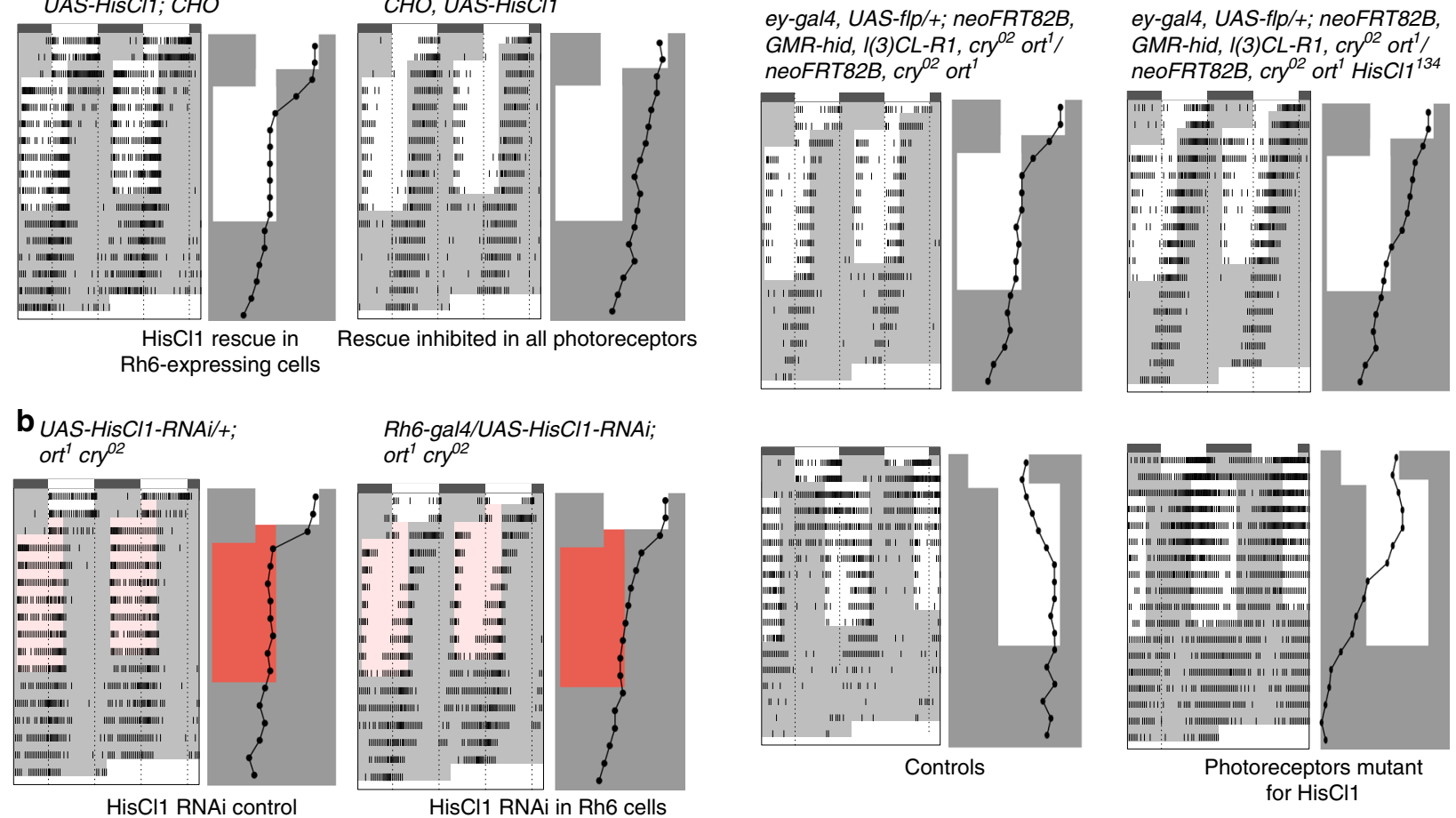

Fig. $4 \mathrm{HisCl1}$ acts in Rh6-expressing photoreceptors to support circadian entrainment. Actograms and phase plots. The experimental design is as described in Fig. 1 legend for advanced LD/RD cycle (a, b, c, top). For delayed LD cycles (c, bottom), the dark phase was lengthened by $8 \mathrm{~h}$ between days 2 and 3. a Expression of HisCl1 specifically in Rh6 photoreceptors with a Rh6-gal4 driver rescues entrainment in CHO triple mutants (left). This rescue is blocked by simultaneous expression of Gal80 in all photoreceptors (right). $\mathbf{b}$ In the absence of Ort and Cry, RNAi knock-down of HisCl1 in Rh6 photoreceptors slows down the synchronization with advanced RD cycles (right), in comparison to controls (left). c In contrast to control flies with HisCl1+ photoreceptors (left), flies with HisCl1734 mutant photoreceptors (right) very poorly synchronize with an advanced LD cycle (top) and fail to synchronize with a delayed LD cycle (bottom)

synchronize with a delayed LD cycle, supporting a critical eye contribution for HisCl1 function. Altogether, the results indicate that photoreceptors are the major site of HisCll expression for synchronization with LD cycles.

Rh6-expressing cells receive multiple photoreceptor inputs. Finally, we investigated the histaminergic input pathways relevant for HisCl1 in Rh6-expressing cells. Flies with HisCl1 only in Rh6 cells synchronized with RD cycles (Fig. 5a, Supplementary Tables 1,2), indicating that Rh6 cells can rely on histaminergic input from photoreceptors expressing Rh1 and/or Rh6 to support entrainment. They synchronized more slowly in the absence of Rh1 (Fig. 5a, Supplementary Fig. 4), suggesting contribution from Rh1 cells. Nevertheless, their ultimate synchronization indicates that inputs from at least some Rh6-expressing photoreceptors contribute to HisCl1 function in Rh6 cells. No synchronization occurred when HisCl1 was restored in Rh6 cells of $\mathrm{CHO}$ mutants in which the compound eye, but not the eyelet, was lost as a consequence of a null mutation in the sine oculis gene (Fig. 5b, Supplementary Tables 1,2 ). Thus, if the Rh6-expressing eyelet is involved in this HisCl1 pathway, it would require inputs from retinal photoreceptors. In addition, genetic ablation of the adult Rh5-expressing cells as well as of the eyelet (see ref. ${ }^{24}$ ) in a $\mathrm{CHO}$ background with $\mathrm{HisCl1}$ rescue in Rh6 cells did not abolish entrainment in $\mathrm{RD}$ cycles (Fig. 5b, Supplementary Table 1, 2). This indicated that the eyelet is not required for HisCll function in Rh6 photoreceptors through Rh1 and/or Rh6 inputs. We then asked whether HisCl1 function in Rh6 cells would require their ability to act as photoreceptors. Since HisCl1 in Rh6-expressing cells could rescue entrainment in the absence of Rh6 (Fig. 5c,
Supplementary Tables 1,2), we can conclude that the interneuron role of Rh6 cells does not require their photoreceptive function. Importantly, efficient synchronization still occurred in the collective absence of Rh1, Rh5, and Rh6 (Fig. 5c, Supplementary Tables 1,2). Although a contribution of the dorsally located Rh2expressing ocelli cannot be totally excluded, this result strongly suggests that R7 photoreceptors, which express UV-sensitive Rh3 or $\mathrm{Rh} 4$, also provide inputs to HisCl1 in Rh6-expressing photoreceptors.

\section{Discussion}

This work reveals that the Cryptochrome-independent entrainment of rest-activity rhythms relies on distinct pathways that are contributed by the two histamine receptors Ort and HisCl1. Whereas Ort mediates circadian entrainment through the optic lobe interneurons that are involved in visual functions, HisCl1 defines a new photoreceptive pathway through Rh6-expressing photoreceptors. Although both receptors mediate synchronization with a shifted LD cycle, it seems likely that the two pathways will show differences in specific light conditions. We could not rescue Ort function with $\mathrm{HisCl1}$ expression in the ort-expressing cells, whereas the Ort could replace HisCl1 in $\mathrm{Rh} 6$ photoreceptors. It is possible that HisCl1 has a lower affinity for histamine with Rh6 cells receiving more neurotransmitter than optic lobe interneurons. Alternatively, interneurons could sufficiently differ from photoreceptors for their physiology or specific receptor-interacting protein content, preventing $\mathrm{HisCl1}$ from working efficiently. HisCl1 downregulation in Rh6 cells slows down synchronization and flies with $\mathrm{HisCl}^{134}$ mutant eyes synchronize very poorly with advanced LD cycles, and fail to 
a Rh6-gal4/UAS-HisCl1; $\mathrm{CHO}$

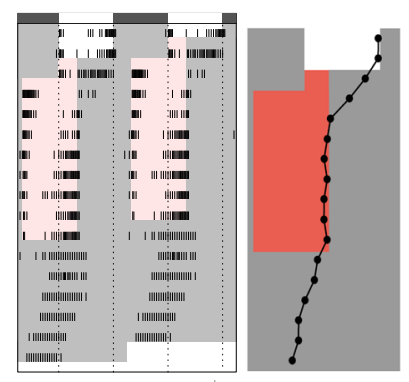

$\mathrm{HisCl} 1$ rescue in Rh6-expressing cells

b

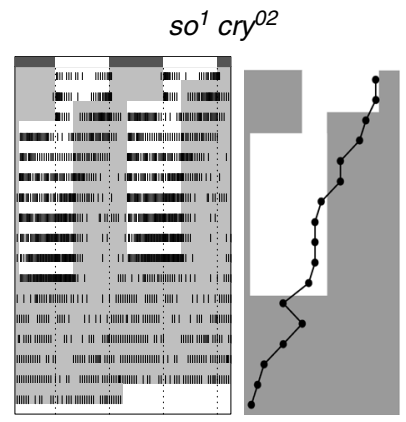

No eye, no Cry
Rh6-gal4/UAS-HisCl1; CHO, ninaE ${ }^{17}$

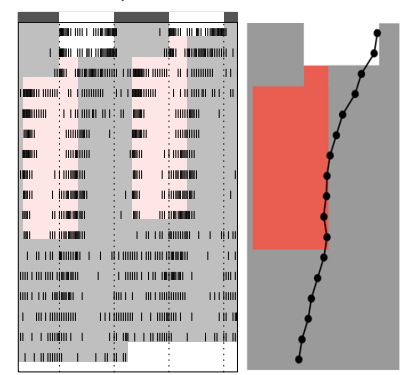

No $\mathrm{Rh} 1, \mathrm{HisCl} 1$ rescue in Rh6-expressing cells

so ${ }^{1}$, Rh6-gal4; $\mathrm{CHO}, \mathrm{UAS}-\mathrm{HisCl1/CHO}$

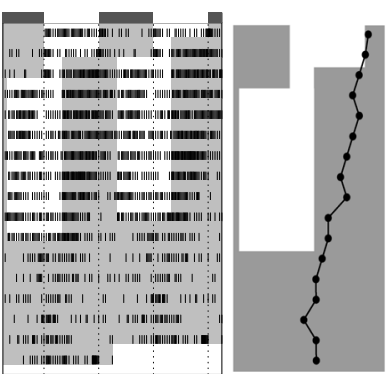

No eye, HisCl1 rescue in Rh6-expressing cells
Rh6-gal4/UAS-HisCl1; $\mathrm{CHO}$, ninaE ${ }^{17} \mathrm{Rh}^{1}$

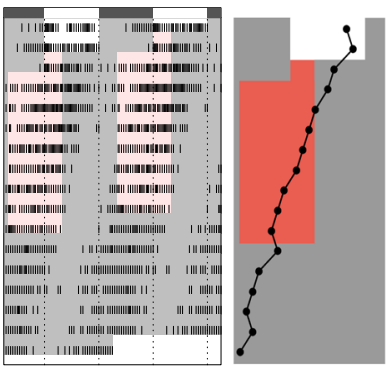

No Rh1, no Rh6, HisCl1 rescue in Rh6-expressing cells

\section{LexAop-HisCl1; Rh6-LexA Rh5-gal4, UAS-dti; CHO}

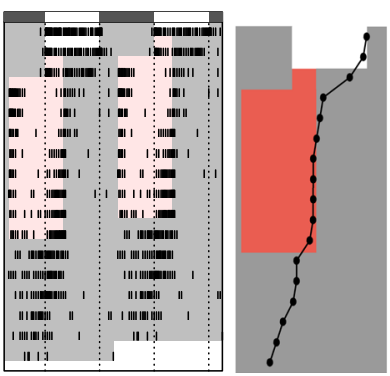

No Rh5-expressing cells, no eyelet, $\mathrm{HisCl} 1$ rescue in Rh6-expressing cells
C $_{\text {Rh6-gal4/UAS-HisCl1; }}$ CHO, ninaE ${ }^{17} R h 6^{1}$

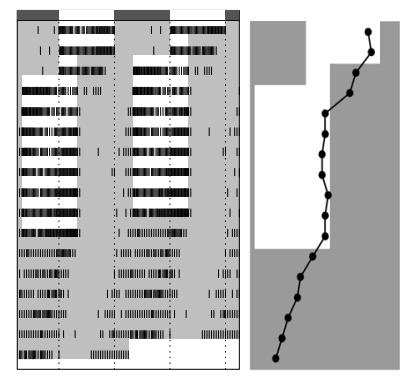

No Rh1, no Rh6, HisCl1 rescue in Rh6-expressing cells

$R h 5^{2}$, Rh6-gal4/Rh5 ${ }^{2}$ UAS-HisCl1; CHO, ninaE ${ }^{17} \mathrm{Rh}^{1}$

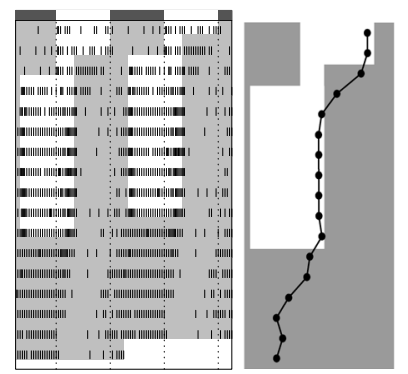

No Rh1, no Rh5, no Rh6, $\mathrm{His} \mathrm{Cl} 1$ rescue

in Rh6-expressing cells

Fig. 5 His Cl1 in Rh6-expressing photoreceptors receive inputs both from outer and inner photoreceptors. a-c Actograms and phase plots. The experimental design is as described in Fig. 1 legend. a The RD phase advance rescue of $\mathrm{CHO}$ flies by HisCl1 expression in Rh6 photoreceptors (left) is slowed down by the absence of Rh1 (ninaE ${ }^{17}$ mutation, center) and is abolished by the absence of both Rh1 and Rh6 (ninaE ${ }^{17}$ Rh6 $^{1}$ genetic background, right). b Flies lacking Cry, compound eyes, and ocelli but retaining the $\mathrm{H}-\mathrm{B}$ eyelet synchronize with advanced LD cycles ( $\mathrm{so}^{7}$; cry $\mathrm{C2}^{2}$ double mutants, left), but do not if HisCl1 expression is restricted to Rh6 photoreceptors (center). $\mathrm{CHO}$ mutants with HisCl1 rescue in Rh6 cells and genetic ablation of the eyelet (in addition to Rh5-expressing photoreceptors) synchronize with advanced RD cycles (right). c The LD phase advance rescue of CHO flies by HisCl1 expression in Rh6 photoreceptors is retained in the absence of either Rh1 and Rh6 (top) or Rh1, Rh5, and Rh6 (bottom)

synchronize with delays. We cannot exclude that nonphotoreceptor cells contribute to HisCl1-dependent entrainment but other pathways appear to have a modest contribution if any.

HisCl1 is expressed in the H-B eyelet, which could thus contribute to this synchronization pathway. However, the cell-killing experiments indicate that $\mathrm{H}-\mathrm{B}$ eyelet is not required for HisCl1mediated synchronization through Rh6 cells. In the recently described color opponency mechanism, retinal R7 cells inhibit R8 and vice versa through HisCl1 expression in the photoreceptors ${ }^{47}$. We suppose that HisCl1-dependent clock synchronization is also mediated by the hyperpolarization of Rh6-expressing cells. How this hyperpolarization interacts with the light-induced depolarization in Rh6 photoreceptors to result in a synchronization message to the clock neurons remains to be understood. Since only Rh6-expressing R8 and not the other inner photoreceptors contribute to this circadian photoreception pathway, Rh6 cells might have specific connections with downstream interneurons. Such specificity has been described for color vision where each of the four inner photoreceptor subtypes connects to a different type of TmY interneuron in the Medulla ${ }^{53}$. We show that HisCl1 expression in Rh6 cells supports synchronization with red light, in the absence of Rh1, indicating that an intra-Rh6-photoreceptor circuit is sufficient. This indicates that Rh6-expressing R8 photoreceptors play a dual photoreceptor/interneuron role in this pathway (Fig. 6). Whether the same individual cells have the two roles is unknown, although the HisCl1-dependent color opponency mechanism suggests that it could be the case. It is also unclear whether all Rh6-expressing R8 photoreceptors or only a fraction of them contribute to circadian synchronization. Our results imply that, in addition to histaminergic neurotransmission, Rh6-expressing photoreceptors can talk to downstream interneurons through histamine-independent neurotransmission (Fig. 6). A recent transcriptomics study indeed revealed the expression of cholinergic markers in R7 and R8 cells, supporting cholinergic transmission in the inner photoreceptors, in addition to histaminergic transmission ${ }^{46}$.

Our data indicate that histaminergic inputs from both outer and inner photoreceptors converge to Rh6 cells to contribute to circadian entrainment. It is possible that some of these inputs rely on Rh7, which seems to be expressed in Rh6-expressing photoreceptors, according to transcriptional reporter data ${ }^{14}$. Putative connections between photoreceptors have been described in Drosophila $47,54,55$ and other insects ${ }^{56,57}$. How R1-6 photoreceptors might be connected to Rh6-expressing R8 cells remains uneasy to understand, but a few putative contacts between presynaptic outer cells and postsynaptic inner cells have been observed in $\mathrm{Musca}^{56}$. The intra-retinal functional connectivity that we report in Drosophila is reminiscent to the circuit logic of circadian entrainment in the mammalian retina, where intrinsically photoreceptive retinal ganglion cells express the melanopsin photopigment in addition to receiving inputs from rods and cones $^{58-60}$. Interestingly, melanopsin appears to share light- 


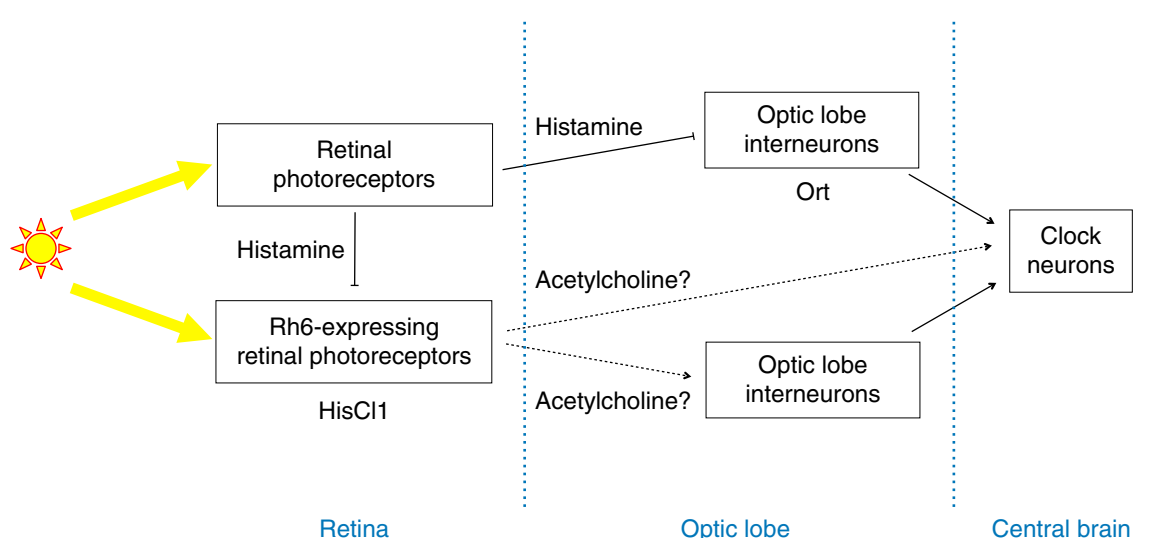

Fig. 6 Model for the retinal input pathways to the brain clock. All retinal photoreceptors receive light inputs and release histamine. Ort-expressing interneurons of the optic lobe receive inhibitory histamine signals from most or all rhodopsin-expressing photoreceptors and transmit light information to the clock neurons through direct or indirect pathways. At least some of the Rh6-expressing photoreceptors express HisCl1 and receive histamine signals from other photoreceptors, in addition to receiving direct light inputs. These Rh6-expressing photoreceptors use a histamine-independent neurotransmission (possibly cholinergic) to transmit light information to the clock neurons. This connection could be either direct or via interneurons of the optic lobe

sensing properties with the rhabdomeric photoreceptors of invertebrates ${ }^{61,62}$. It has been shown that the mammalian circadian clock can synchronize with day-night cycles by tracking light color changes in addition to light intensity changes ${ }^{63,64}$. It will be interesting to investigate the possible contribution of the dual function of Rh6-expressing photoreceptors to integrating different color cues into the retinal information that is sent to the clock.

\section{Methods}

Fly stocks. The HisCl1::egfp, ey-rfp fosmid (see ref. ${ }^{65}$ ) contains a large genomic DNA fragment encompassing the HisCl1 gene (VDRC 318735). Two different HisCl1-gal4 constructs were used, which slightly differ in the length of the 5 ' sequences driving gal4 expression ${ }^{41,42}$. A list of all stocks is provided in the accompanying supplementary information.

Generation of transgenic lines. To produce UAS-HisCll flies, we prepared mRNA from Canton $S$ flies, amplified the open reading frame (ORF) of the HisCll gene with reverse transcription (RT)-PCR. The following primers were used for RTPCR: forward, 5'-CACCATGCAAAGCCCAACTAGCAAATT- 3 ' and reverse, 5'TCATAGGAACGTTGTCCAATAG-3'. The resulting complementary DNA (cDNA) was cloned into an entry TOPO vector (pENTR/D-TOPO, Invitrogen). The UAS-HisCl1 transgene was generated by recombining the HisCl1 ORF into a destination pTHW vector (Drosophila Gateway System) in frame with a Nterminal 3xHA epitope tag. Then this construct was introduced into fly by standard insertion of a P-element (Fly Facility, France). One insertion on the second chromosome and one on the third chromosome were used in this study. Both rescue entrainment of $\mathrm{CHO}$ mutants when driven by tim-gal4. LexAop-HisCll flies were generated by amplifying the HA-HisCll coding region from the $w$; UAS-HAHisCl1 line with forward primer 5'-tatCTCGAGATGGATCTCCACCGCGGT-3' and reverse primer $5^{\prime}$-aaTCTAGATCATAGGAACGTTGTCCAATAGACA-3'. The plasmid pJFRC19-13XLexAop2-IVS-myr::gfp (Addgene \#26224) was digested with XhoI and XbaI to excise the Myr::gfp sequence and replace it with HA-HisCl1 amplicon previously digested with XhoI and XbaI. After sequencing the coding region, the construct was introduced inside the $\mathrm{X}$ chromosome attP8 docking site doing a phiC31 integrase-mediated transformation (BestGene).

Generation of $\mathbf{H i s} \mathbf{C l} \mathbf{1}$ mutant eye clones. Flies with $\mathrm{HisCl1}^{134}$ eye clones were generated by using eyeless-driven flippase recognition target (FRT)-mediated mitotic recombination ${ }^{66}$. The stocks required for generation of the somatic clones of the eye were obtained from Bloomington Drosophila Stock Center (BDSC). BDSC\#5253 containing eyeless-gal4, UAS-flp on the second chromosome, and neoFRT ${ }^{82 B}$ GMR-hid, l(3)CL-R ${ }^{1}$ on the third chromosome was crossed with $c r y^{02}$ ort ${ }^{1}$ flies to put the different transgenes in a double mutant background by genetic recombination. BDSC $\# 2035$ containing neoFRT $T^{82 B}$ on the third chromosome was used to put this transgene in a $\mathrm{cry}^{02} \mathrm{ort}^{1}$ background or in a $\mathrm{cry}^{02}$, ort ${ }^{1}, \mathrm{Hiscll}^{134}$ background. The three lines were PCR-controlled for the presence of neomycinR, $c r y^{02}$, ort ${ }^{1}$, and Hiscl $1^{134}$ when applicable. The ey-gal4-induced FRT recombination produced flies with a close to wild-type eye morphology in the presence of GMRhid, l(3)CL-R $R^{1}$, indicating that vast majority of photoreceptors were homozygous for the Hiscl1 ${ }^{134}$ mutation. The final genotypes were $w$; ey-gal4, UAS-flp/+; neoFRT82B, GMR-hid, l(3)CL-R $R^{1}$ cry ${ }^{02}$ ort $^{1} /$ neoFRT82B, cry $^{02}$ ort $^{1}$ (control) and $w$; ey-gal4, UAS-flp/+; neoFRT ${ }^{22 B}$, GMR-hid, l(3)CL-R ${ }^{1}$, cry $^{02}$ ort $^{1} /$ neoFRT $T^{82 B}$, cry $^{02}$ ort $^{1}$ Hiscll $^{134}$ (mutant).

Behavioral analysis. Experiments were conducted with 3-5-day-old males placed in Drosophila activity monitors (TriKinetics) as described in ref. ${ }^{29}$. In all experiments, flies were first entrained for 2 days under $\mathrm{LD}+\mathrm{T}^{\circ} \mathrm{C}$ cycles $12 \mathrm{~h}$ light $\left(25^{\circ} \mathrm{C}\right)-12 \mathrm{~h}$ dark $\left(20^{\circ} \mathrm{C}\right)$. Light was provided by light-emitting diodes (LEDs; $400 \mathrm{~nm}-680 \mathrm{~nm}$ at $\sim 2000$ lux). For standard (high light) LD (light-dark) phase advances, $\mathrm{T}^{\circ} \mathrm{C}$ was kept constant $\left(25^{\circ} \mathrm{C}\right)$ from the beginning of the day 3 light phase until the end of the experiment and the day 3 light phase was shortened by $8 \mathrm{~h}$, followed by LD 8 cycles (LED source, $400 \mathrm{~nm}-680 \mathrm{~nm}$ at $\sim 2000$ lux), then constant darkness (6 DD cycles). For low light LD advances, the 8 advanced LD cycles were done with dim light (LED source, $325 \mathrm{~nm}-625 \mathrm{~nm}$ at $\sim 5$ lux). For RD (red light-dark) advances (LED source, $590 \mathrm{~nm}-680 \mathrm{~nm}$ at $\sim 250$ lux), short-night or short-day protocols were used and gave the same results. For short night (Fig. 1 and Supplementary Fig. 1$), \mathrm{T}^{\circ} \mathrm{C}$ was kept constant $\left(25^{\circ} \mathrm{C}\right)$ from the beginning of the day 2 light phase until the end of the experiment. Between days 2 and 3, the dark phase was shortened by $8 \mathrm{~h}$, followed by RD 8 cycles, then constant darkness (6 DD cycles). For short days (Figs. 3 and 4 ), $\mathrm{T}^{\circ} \mathrm{C}$ was kept constant $\left(25^{\circ} \mathrm{C}\right)$ from the beginning of the day 3 light phase until the end of the experiment. The day 3 light phase was shortened by $8 \mathrm{~h}$ and red light was used instead of white light, followed by RD 8 cycles, then constant darkness (6 DD cycles). For standard (high light) LD phase delays, $\mathrm{T}^{\circ} \mathrm{C}$ was kept constant $\left(25^{\circ} \mathrm{C}\right)$ from the beginning of the day 2 light phase until the end of the experiment. Between days 2 and 3, the dark phase was lengthened by $8 \mathrm{~h}$ followed by LD 8 cycles $(400 \mathrm{~nm}-680 \mathrm{~nm}$ LEDs at 2000 lux), then constant darkness (6 DD cycles). Data analysis was done with the FaasX 1.21 software, which is derived from the Brandeis Rhythm Package ${ }^{67,68}$. FaasX runs on Apple Macintosh OSX and is freely available (http://neuro-psi.cnrs. fr/spip.php?article298\&lang=en). Bin size was $30 \mathrm{~min}$. The data are shown as average double-plotted actograms from $n$ flies. All behavioral experiments were done at least three times (except when indicated) with similar results. The statistical analysis of the phase data was done as follows. For each day, the ZT that corresponds to the peak value of evening activity (second half of the day) in $\mathrm{LD} / \mathrm{RD}$ cycles was determined from the averaged activity data ( $n$ flies). These values were used to generate phase plots that are shown next to the corresponding actograms to better visualize the daily phase during all experiments. Entrainment was then estimated by comparing the peak ZT values of the last 5 days of the shifted LD/RD regime to those of Canton S controls. A genotype was considered entrained when this series of five values was not significantly different from the control one in a one-way analysis of variance (ANOVA) Dunnett's multiple comparisons test (GraphPad Prism 7 software). Results of the ANOVA are shown in Supplementary Table 2. Of course, we cannot exclude that genotypes that are not synchronized during the 8-day interval would do it over a longer interval, or in another experimental paradigm. The period of free-running rhythms in DD was estimated by $x^{2}$ periodogram analysis from the last 5 days of data with the following criteria for rhythmic flies (filter ON): power $\geq 20$, width $\geq 2 \mathrm{~h}$. Power and width correspond to the height and width of the periodogram peak, respectively, and give the significance of the calculated period.

Immunolabelings. Brains with retinas were dissected, fixed, and labeled as previously described ${ }^{69}$. Briefly, we dissected brains with the retinas attached to the 
optic lobes and incubation with the primary antibodies anti-GFP (chicken, Invitrogen) and anti-Chaoptin (mouse, clone 24B10, gift from A. Hofbauer), was done at least $48 \mathrm{~h}$ at $4{ }^{\circ} \mathrm{C}$, followed by PBT (phosphate buffer saline (PBS) $+0.1 \%$ Tween-20) washes and incubation with secondary antibodies, anti-chicken Alexa647 conjugated (1:1000) and Alexa-488 conjugated (1:1000) and anti-mouse FP547-conjugated $(1: 1000)$ for at least $24 \mathrm{~h}$ at $4{ }^{\circ} \mathrm{C}$. The samples were imaged with a Zeiss AxioImager microscope with an ApoTome structured illumination module.

In situ hybridization. In situ hybridization was performed with the RNAscope Multiplex Fluorescent Reagent Kit v2 (ACD Bio) ${ }^{70,71}$ using a protocol optimized for whole mount Drosophila adult retina staining. Unless specified otherwise, the solutions supplied with the kit were used. The retinas with or without the attached laminas were dissected in PBS as in ref. ${ }^{72}$, fixed in paraformaldehyde $3.7 \%$ for $1 \mathrm{~h}$ at room temperature $\left(\mathrm{RT}^{\circ} \mathrm{C}\right)$ and washed with PBT. The tissue was treated with $3 \%$ hydrogen peroxide $\left(3 \% \mathrm{H}_{2} \mathrm{O}_{2}\right)$ for $20 \mathrm{~min}$ at $\mathrm{RT}^{\circ} \mathrm{C}$. The target retrieval treatment was done for $2 \mathrm{~min}$ at $100{ }^{\circ} \mathrm{C}$ in $1 \times$ Target Retrieval solution. Retinas were then treated with undiluted Protease IV for $30 \mathrm{~min}$ at RT. Each of the preceding steps was followed by two 10 min washes in PBT $+1 \%$ bovine serum albumin. Retinas were transferred to probe diluent solution. The probes were warmed to $40{ }^{\circ} \mathrm{C}$ and added to the samples: HisCl1 probe (Cat. No. 300031-C3 created by ACD Bio for the present study) was diluted (1:50 in probe diluent), whereas the ort probe (Cat. No. 435481, ACD Bio) was undiluted. After an overnight incubation at $40^{\circ} \mathrm{C}$, the samples were washed twice $10 \mathrm{~min}$ with $1 \times$ Wash Buffer and incubated with 2-3 drops of RNAscope ${ }^{\oplus}$ Multiplex FL v2 at $40^{\circ} \mathrm{C}$ for $30 \mathrm{~min}$ and then washed as above. These steps were repeated with RNAscope ${ }^{\circledast}$ Multiplex FL v2 Amp 2 and then RNAscope ${ }^{\circledast}$ Multiplex FL v2 Amp 3 incubations. The samples for ort and negative control stains were incubated with RNAscope ${ }^{\oplus}$ Multiplex FL v2 HRP-C1, and for $\mathrm{HisCl}$ stains with RNAscope ${ }^{\circledast}$ Multiplex FL v2 HRP-C3 for 15 min at $40{ }^{\circ} \mathrm{C}$, followed by washes as before. Finally, the samples were incubated for $30 \mathrm{~min}$ at $40^{\circ} \mathrm{C}$ with Opal 520 (Perkin Elmer, FP1487001KT, 1:2000 dilution) for ort and negative control probes and Opal 650 (Perkin Elmer, FP1496001KT, 1:2000 dilution) for $\mathrm{His} C l 1$ probe. The samples were washed as above and incubated overnight at $4{ }^{\circ} \mathrm{C}$ with anti-Rh6 antibody (rabbit, $\left.1: 1000\right)^{73}$, washed in PBT, and incubated with a secondary Alexa555-conjugated donkey anti-rabbit antibody (Invitrogen, Cat. No. $1891766,1: 500)$ for $3 \mathrm{~h}$ at $\mathrm{RT}^{\circ} \mathrm{C}$. Retinas were mounted on glass slides with SlowFade ${ }^{\mathrm{TM}}$ Gold antifade reagent (Invitrogen) as in ref. ${ }^{72}$. The samples were imaged with SP8 Leica Confocal Microscope.

\section{Data availability}

The datasets generated and analyzed during the current study are available from the corresponding author on reasonable request.

Received: 27 February 2018 Accepted: 19 December 2018

Published online: 16 January 2019

\section{References}

1. Dubowy, C. \& Sehgal, A. Circadian rhythms and sleep in Drosophila melanogaster. Genetics 205, 1373-1397 (2017).

2. Helfrich-Förster, C., Winter, C., Hofbauer, A., Hall, J. C. \& Stanewsky, R. The circadian clock of fruit flies is blind after elimination of all known photoreceptors. Neuron 30, 249-261 (2001).

3. Yoshii, T., Hermann-Luibl, C. \& Helfrich-Förster, C. Circadian light-input pathways in Drosophila. Commun. Integr. Biol. 9, e1102805 (2016).

4. Stanewsky, R. et al. The cryb mutation identifies cryptochrome as a circadian photoreceptor in Drosophila. Cell 95, 681-692 (1998).

5. Emery, P., So, W. V., Kaneko, M., Hall, J. C. \& Rosbash, M. CRY, a Drosophila clock and light-regulated cryptochrome, is a major contributor to circadian rhythm resetting and photosensitivity. Cell 95, 669-679 (1998).

6. Emery, P. et al. Drosophila CRY is a deep brain circadian photoreceptor. Neuron 26, 493-504 (2000).

7. Klarsfeld, A. et al. Novel features of cryptochrome-mediated photoreception in the brain circadian clock of Drosophila. J. Neurosci. 24, 1468-1477 (2004).

8. Yoshii, T., Todo, T., Wulbeck, C., Stanewsky, R. \& Helfrich-Forster, C. Cryptochrome is present in the compound eye and a subset of Drosophila's clock neurons. J. Comp. Neurol. 508, 952-966 (2008).

9. Helfrich-Förster, C. et al. The extraretinal eyelet of Drosophila: development, ultrastructure and putative circadian function. J. Neurosci. 22, 9255-9266 (2002).

10. Rieger, D., Stanewsky, R. \& Helfrich-Förster, C. Cryptochrome, compound eyes, $\mathrm{H}-\mathrm{B}$ eyelets and ocelli play different roles in the entrainment and masking pathway of the locomotor activity rhythm in the fruit fly Drosophila melanogaster. J. Biol. Rhythms 18, 377-391 (2003).

11. Umezaki, Y. \& Tomioka, K. Behavioral dissection of the Drosophila circadian multioscillator system that regulates locomotor rhythms. Zool. Sci. 25, 1146-1155 (2008).
12. Ni, J. D., Baik, L. S., Holmes, T. C. \& Montell, C. A rhodopsin in the brain functions in circadian photoentrainment in Drosophila. Nature 545, 340-344 (2017).

13. Grebler, R. et al. Drosophila Rhodopsin 7 can partially replace the structural role of Rhodopsin 1, but not its physiological function. J. Comp. Physiol. A Neuroethol. Sens. Neural Behav. Physiol. 203, 649-659 (2017).

14. Kistenpfennig, C. et al. A new rhodopsin influences light-dependent daily activity patterns of fruit flies. J. Biol. Rhythms 32, 406-422 (2017).

15. Picot, M., Cusumano, P., Klarsfeld, A., Ueda, R. \& Rouyer, F. Light activates output from evening neurons and inhibits output from morning neurons in the Drosophila circadian clock. PLoS Biol. 5, e315 (2007).

16. Zhang, Y., Liu, Y., Bilodeau-Wentworth, D., Hardin, P. E. \& Emery, P. Light and temperature control the contribution of specific DN1 neurons to Drosophila circadian behavior. Curr. Biol. 20, 600-605 (2010).

17. Chatterjee, A. et al. Reconfiguration of a multi-oscillator network by light in the Drosophila circadian clock. Curr. Biol. 28, 2007-2017 (2018).

18. Salcedo, E. et al. Blue- and green-absorbing visual pigments of Drosophila: ectopic expression and physiological characterization of the R8 photoreceptor cell-specific Rh5 and Rh6 rhodopsins. J. Neurosci. 19, 10716-10726 (1999).

19. Behnia, R. \& Desplan, C. Visual circuits in flies: beginning to see the whole picture. Curr. Opin. Neurobiol. 34, 125-132 (2015).

20. Renn, S. C., Park, J. H., Rosbash, M., Hall, J. C. \& Taghert, P. H. A pdf neuropeptide gene mutation and ablation of PDF neurons each cause severe abnormalities of behavioral circadian rhythms in Drosophila. Cell 99, 791-802 (1999).

21. Hofbauer, A. \& Buchner, E. Does Drosophila have seven eyes? Naturwissenschaften 76, 335-336 (1989).

22. Yasuyama, K. \& Meinertzhagen, I. A. Extraretinal photoreceptors at the compound Eye's posterior margin in drosophila melanogaster. J. Comp. Neurol. 412, 193-202 (1999).

23. Malpel, S., Klarsfeld, A. \& Rouyer, F. Larval optic nerve and adult extraretinal photoreceptors sequentially associate with the clock neurons during Drosophila brain development. Development 129, 1443-1453 (2002).

24. Sprecher, S. G. \& Desplan, C. Switch of rhodopsin expression in terminally differentiated Drosophila sensory neurons. Nature 454, 533-537 (2008).

25. Pollock, J. A. \& Benzer, S. Transcript localization of four opsin genes in the three visual organs of Drosophila; RH2 is ocellus specific. Nature 333, 779-782 (1988).

26. Hanai, S., Hamasaka, Y. \& Ishida, N. Circadian entrainment to red light in Drosophila: requirement of Rhodopsin 1 and Rhodopsin 6. Neuroreport 19, 1441-1444 (2008)

27. Hardie, R. C. \& Raghu, P. Visual transduction in Drosophila. Nature 413, 186-193 (2001).

28. Montell, C. Drosophila visual transduction. Trends Neurosci. 35, 356-363 (2012).

29. Saint-Charles, A. et al. Four of the six Drosophila rhodopsin-expressing photoreceptors can mediate circadian entrainment in low light. J. Comp. Neurol. 524, 2828-2844 (2016).

30. Szular, J. et al. Rhodopsin 5- and rhodopsin 6-mediated clock synchronization in Drosophila melanogaster is independent of retinal phospholipase C-beta signaling. J. Biol. Rhythms 27, 25-36 (2012).

31. Ogueta, M., Hardie, R. C. \& Stanewsky, R. Non-canonical phototransduction mediates synchronization of the Drosophila melanogaster circadian clock and retinal light responses. Curr. Biol. 28, 1725-1735.e3 (2018).

32. Pollack, I. \& Hofbauer, A. Histamine-like immunoreactivity in the visual system and brain of Drosophila melanogaster. Cell Tissue Res. 266, 391-398 (1991).

33. Hamasaka, Y. \& Nassel, D. R. Mapping of serotonin, dopamine, and histamine in relation to different clock neurons in the brain of Drosophila. J. Comp. Neurol. 494, 314-330 (2006).

34. Muraro, N. I. \& Ceriani, M. F. Acetylcholine from visual circuits modulates the activity of arousal neurons in Drosophila. J. Neurosci. 35, 16315-16327 (2015).

35. Schlichting, M. et al. A neural network underlying circadian entrainment and photoperiodic adjustment of sleep and activity in Drosophila. J. Neurosci. 36, 9084-9096 (2016).

36. Gengs, C. et al. The target of Drosophila photoreceptor synaptic transmission is a histamine-gated chloride channel encoded by ort (hclA). J. Biol. Chem. 277, 42113-42120 (2002).

37. Gisselmann, G., Pusch, H., Hovemann, B. T. \& Hatt, H. Two cDNAs coding for histamine-gated ion channels in D. melanogaster. Nat. Neurosci. 5, 11-12 (2002).

38. Witte, I., Kreienkamp, H. J., Gewecke, M. \& Roeder, T. Putative histaminegated chloride channel subunits of the insect visual system and thoracic ganglion. J. Neurochem. 83, 504-514 (2002).

39. Zheng, Y. et al. Identification of two novel Drosophila melanogaster histamine-gated chloride channel subunits expressed in the eye. J. Biol. Chem. 277, 2000-2005 (2002). 
40. Rister, J. et al. Dissection of the peripheral motion channel in the visual system of Drosophila melanogaster. Neuron 56, 155-170 (2007).

41. Gao, S. et al. The neural substrate of spectral preference in Drosophila. Neuron 60, 328-342 (2008).

42. Pantazis, A. et al. Distinct roles for two histamine receptors (hclA and hclB) at the Drosophila photoreceptor synapse. J. Neurosci. 28, 7250-7259 (2008).

43. Hong, S. T. et al. Histamine and its receptors modulate temperaturepreference behaviors in Drosophila. J. Neurosci. 26, 7245-7256 (2006).

44. Lin, T. Y. et al. Mapping chromatic pathways in the Drosophila visual system. J. Comp. Neurol. 524, 213-227 (2016).

45. Tan, L. et al. Ig superfamily ligand and receptor pairs expressed in synaptic partners in Drosophila. Cell 163, 1756-1769 (2015).

46. Davis, F. P. et al. A genetic, genomic, and computational resource for exploring neural circuit function. Preprint at BioRxiv https://doi.org/10.1101/ 385476 (2018).

47. Schnaitmann, C. et al. Color processing in the early visual system of Drosophila. Cell 172, 318-330.e18 (2018).

48. Kaneko, M. \& Hall, J. C. Neuroanatomy of cells expressing clock genes in Drosophila: transgenic manipulation of the period and timeless genes to mark the perikarya of circadian pacemaker neurons and their projections. J. Comp. Neurol. 422, 66-94 (2000).

49. Lai, S. L. \& Lee, T. Genetic mosaic with dual binary transcriptional systems in Drosophila. Nat. Neurosci. 9, 703-709 (2006).

50. Helfrich-Förster, C. \& Homberg, U. Pigment-dispersing hormoneimmunoreactive neurons in the nervous system of wild-type Drosophila melanogaster and of several mutants with altered circadian rhythmicity. J. Comp. Neurol. 337, 177-190 (1993).

51. Gummadova, J. O., Coutts, G. A. \& Glossop, N. R. Analysis of the Drosophila clock promoter reveals heterogeneity in expression between subgroups of central oscillator cells and identifies a novel enhancer region. J. Biol. Rhythms 24, 353-367 (2009).

52. Papatsenko, D., Sheng, G. \& Desplan, C. A new rhodopsin in R8 photoreceptors of Drosophila: evidence for coordinate expression with Rh3 in R7 cells. Development 124, 1665-1673 (1997).

53. Jagadish, S., Barnea, G., Clandinin, T. R. \& Axel, R. Identifying functional connections of the inner photoreceptors in Drosophila using tango-trace. Neuron 83, 630-644 (2014).

54. Takemura, S. Y., Lu, Z. \& Meinertzhagen, I. A. Synaptic circuits of the Drosophila optic lobe: the input terminals to the medulla. J. Comp. Neurol. 509, 493-513 (2008).

55. Wardill, T. J. et al. Multiple spectral inputs improve motion discrimination in the Drosophila visual system. Science 336, 925-931 (2012).

56. Shaw, S. R., Fröhlich, A. \& Meinertzhagen, I. A. Direct connections between the R7/8 and R1-6 photoreceptor subsystems in the dipteran visual system. Cell Tissue Res. 257, 295-302 (1989).

57. Takemura, S. Y. \& Arikawa, K. Ommatidial type-specific interphotoreceptor connections in the lamina of the swallowtail butterfly, Papilio xuthus. J. Comp. Neurol. 494, 663-672 (2006).

58. Hattar, S., Liao, H. W., Takao, M., Berson, D. M. \& Yau, K. W. Melanopsincontaining retinal ganglion cells: architecture, projections, and intrinsic photosensitivity. Science 295, 1065-1070 (2002).

59. Provencio, I., Rollag, M. D. \& Castrucci, A. M. Photoreceptive net in the mammalian retina. This mesh of cells may explain how some blind mice can still tell day from night. Nature 415, 493 (2002).

60. Lucas, R. J. Mammalian inner retinal photoreception. Curr. Biol. 23, R125-R133 (2013).

61. Hankins, M. W., Peirson, S. N. \& Foster, R. G. Melanopsin: an exciting photopigment. Trends Neurosci. 31, 27-36 (2008).

62. Díaz, N. M., Morera, L. P. \& Guido, M. E. Melanopsin and the non-visual photochemistry in the inner retina of vertebrates. Photochem. Photobiol. 92, 29-44 (2016).

63. Walmsley, L. et al. Colour as a signal for entraining the mammalian circadian clock. PLoS Biol. 13, e1002127 (2015).

64. Spitschan, M., Lucas, R. J. \& Brown, T. M. Chromatic clocks: color opponency in non-image-forming visual function. Neurosci. Biobehav. Rev. 78, 24-33 (2017).

65. Sarov, M. et al. A genome-wide resource for the analysis of protein localisation in Drosophila. eLife 5, e12068 (2016).
66. Stowers, R. S. \& Schwarz, T. L. A genetic method for generating drosophila eyes composed exclusively of mitotic clones of a single genotype. Genetics 152, $1631-1639$ (1999)

67. Hamblen, M. et al. Germ-line transformation involving DNA from the period locus in Drosophila melanogaster: overlapping genomic fragments that restore circadian and ultradian rhythmicity to per0 and per-mutants. J. Neurogenet. 3 , 249-291 (1986).

68. Klarsfeld, A., Leloup, J. C. \& Rouyer, F. Circadian rhythms of locomotor activity in Drosophila. Behav. Process. 64, 161-175 (2003).

69. Cusumano, P. et al. PDF-modulated visual inputs and Cryptochrome define diurnal behavior in Drosophila. Nat. Neurosci. 12, 1427-1433 (2009).

70. Wang, F. et al. RNAscope: a novel in situ RNA analysis platform for formalinfixed, paraffin-embedded tissues. J. Mol. Diagn. 14, 22-29 (2012).

71. Wang, X. \& Page-McCaw, A. Wnt6 maintains anterior escort cells as an integral component of the germline stem cell niche. Development 145, pii: dev158527 (2018).

72. Hsiao, H. Y. et al. Dissection and immunohistochemistry of larval, pupal and adult Drosophila retinas. J. Vis. Exp. 69, 4347 (2012).

73. Tahayato, A. et al. Otd/Crx, a dual regulator for the specification of ommatidia subtypes in the Drosophila retina. Dev. Cell 5, 391-402 (2003).

\section{Acknowledgements}

We thank the Bloomington Drosophila Stock Center and the Vienna Drosophila Research Center (VDRC) for fly stocks, M. Boudinot for the FaasX software, as well as A. Chatterjee and J. Carcaud for discussions and insightful comments on the manuscript. D.V. acknowledges funding from European Union 7th Framework Program (Marie Curie Career Integration Grant). F.R. acknowledges funding from Agence Nationale de la Recherche (ClockEye and TEFOR grants), Fondation pour la Recherche Médicale (Equipe FRM grant), and European Union 6th (EUCLOCK Integrated Project) and 7th (INsecTIME Initial Training Network) Framework Programs. D.V. and F.R. are supported by Institut National de la Santé et de la Recherche Médicale (INSERM).

\section{Author contributions}

F.A., A.S.-C., C.M-V. and F.R. designed the project. F.A., A.S.-C., C.M.-V., B.M., S.G. and D.V. designed and performed the experiments. F.R. wrote the manuscript with input from F.A., A.S.-C., C.M-V., and D.V.

\section{Additional information}

Supplementary Information accompanies this paper at https://doi.org/10.1038/s41467018-08116-7.

Competing interests: The authors declare no competing interests.

Reprints and permission information is available online at http://npg.nature.com/ reprintsandpermissions/

Journal peer review information: Nature Communications thanks the anonymous reviewers for their contribution to the peer review of this work. Peer reviewer reports are available.

Publisher's note: Springer Nature remains neutral with regard to jurisdictional claims in published maps and institutional affiliations.

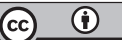

Open Access This article is licensed under a Creative Commons Attribution 4.0 International License, which permits use, sharing, adaptation, distribution and reproduction in any medium or format, as long as you give appropriate credit to the original author(s) and the source, provide a link to the Creative Commons license, and indicate if changes were made. The images or other third party material in this article are included in the article's Creative Commons license, unless indicated otherwise in a credit line to the material. If material is not included in the article's Creative Commons license and your intended use is not permitted by statutory regulation or exceeds the permitted use, you will need to obtain permission directly from the copyright holder. To view a copy of this license, visit http://creativecommons.org/licenses/by/4.0/.

(C) The Author(s) 2019 